\title{
Comportamento espectral de espécies utilizadas em arborização urbana ${ }^{1}$
}

\author{
Leticia Daiane Pedrali²; Juliana Marchesan³; Túlio Barroso Queiroz ${ }^{4}$; Talita Baldin ${ }^{5}$; Rita dos Santos \\ Sousa $^{6}$; Fábio de Jesus Batista ${ }^{7}$; Rudiney Soares Pereira ${ }^{8}$
}

Resumo: O objetivo desta pesquisa foi caracterizar e comparar o comportamento espectral de seis espécies arbóreas presentes na Universidade Federal de Santa Maria/RS. As espécies estudadas foram Eucalyptus sp., Ficus benjamina, Inga marginata, Schinus terebinthifolius, Psidium cattleyanum e Handroanthus chrysotrichus. Foram coletadas aleatoriamente sete folhas sadias por espécie, posteriormente analisadas pelo espectrorradiômetro FieldSpec®3, no intervalo de 350 a $2.500 \mathrm{~nm}$. As reflectâncias médias foram utilizadas no cálculo de 61 índices de vegetação. A partir do matriz índice x espécie realizou-se análise de componentes principais. As diferenças entre os comportamentos espectrais das espécies, por bandas do sensor WorldView-2, foram testadas por meio de análise de variância (Tukey). As análises estatísticas foram realizadas no programa R. A análise de agrupamento (método Ward), considerando a distância euclidiana como medida de dissimilaridade, foi realizada com o PC-ORD 6.0. A obtenção e o processamento dos dados espectrais foram realizados nos programas ASD ViewSpecPro 4.05 e SAMS 3.2. O primeiro componente abrangeu 90,94\% da variância dos dados. Como resultado, observouse que a banda com menor relação entre o agrupamento e as diferenças das médias por espécie foi a do Vermelho, enquanto as bandas do Costal, Azul, Verde, Amarelo e Vermelho obtiveram comportamentos semelhantes, com diferenciação das espécies $F$. benjamina (exceto na banda do Verde), I. marginata (Verde e Amarelo), e P. cattleyanum (Verde). Na banda RedEdge não houve distinção entre espécies. As diferenças de refletâncias foram mais evidentes nas bandas Infravermelho Próximo 1 e 2. Os Índices de Vegetação Otimizada 1 e Razão Simples 8 foram os mais indicados nos estudos das espécies.

Palavras - chave: Índice de vegetação; Espectroscopia; Sensoriamento remoto.

\section{Spectral behavior of species used in urban afforestation}

\begin{abstract}
The aim of this research was to characterize and measure the spectral behavior of six tree species grown at the Federal University of Santa Maria/RS. The studied species were Eucalyptus sp., Ficus benjamina, Inga marginata, Schinus terebinthifolius, Psidium cattleyanum and Handroanthus chrysotrichus. Were randomly collected seven healthy leaves for each specie, andanalyzed with FieldSpec3 Spectroradiometer, in the wavelength range from 350 to $2.500 \mathrm{~nm}$. Were calculated 61 vegetation indices based on the mean average reflectances. Was applied a principal component analysis based on array $\mathrm{x}$ species matrice. The differences between the spectral behavior of the species were tested with a variance analysis (Tukey) by each spectral band of the Worldview 2 satellite sensor. The statistical analyses were performed with R software. Hierarchical clustering analysis was performed with PC-ORD 6.0 taking into account the Euclidean distance as measure of dissimilarity. To obtain and process spectral data were used ASD ViewSpecPro 4.05 and SAMS 3.2 softwares. The first component comprehended $90.94 \%$ of the variance for the dataset. The lowest ratio between the group and the average differences per specie was in the Red band. The Coastal, Blue, Green, Yellow and Red bands obtained similar behaviors, with differences in the species $F$. benjamina (except Green's band), I. marginata (Green and Yellow), and $P$. cattleyanum (Green). The Red Edge band presented no distinction between species. Reflectance differences were more visible in the Near Infrared 1 and 2 bands. Optimized Vegetation Index 1 and Simple Ratio 8 were the most effective indices to study the species.
\end{abstract}

Keywords: Vegetation index; Spectroscopy; Remote sensing.

\footnotetext{
${ }^{1}$ Recebido em 12.07.2016 e aceito para publicação como artigo científico em 26.10.2016.

${ }^{2}$ Engenheira Florestal, mestranda do Programa de Pós Graduação em Engenharia Florestal, Universidade Federal de Santa Maria, E-mail: $<$ lepedrali@yahoo.com.br>.

${ }^{3}$ Engenheira Florestal, mestranda do Programa de Pós Graduação em Engenharia Florestal, Universidade Federal de Santa Maria, E-mail: <marchesan.ju@gmail.com>.

${ }^{4}$ Engenheiro Florestal, mestrando do Programa de Pós Graduação em Engenharia Florestal, Universidade Federal de Santa Maria, E-mail: <tulioeng.florestal@gmail.com>.

${ }^{5}$ Engenheira Florestal, M.Sc. em Engenharia Florestal, Doutoranda do Programa de Pós Graduação em Engenharia Florestal, Universidade Federal de Santa Maria, E-mail: <baldintalita@gmail.com>.

${ }^{6}$ Engenheira Biofísica, M.Sc. em Engenharia Florestal, Doutoranda do Programa de Pós Graduação em Engenharia Florestal, Universidade Federal de Santa Maria, E-mail: <ritasousa.ufsm@gmail.com>.

${ }^{7}$ Engenheiro Florestal, M.Sc. em botânica, Doutorando do Programa de Pós Graduação em Engenharia Florestal, Universidade Federal de Santa Maria, E-mail: <fabiodejesusbatista@gmail.com>.

${ }^{8}$ Engenheiro Florestal, Dr., Professor Titular do Departamento de Ciências Florestais, Universidade Federal de Santa Maria. E-mail: <rudiney.s.pereira@gmail.com>.
} 


\section{Introdução}

A arborização urbana apresenta um importante papel na atenuação da radiação solar de espaços de uso público (BASSO e CORREA, 2014). Estudos, que relacionem a vegetação urbana com a sanidade do vegetal, possibilitam maior compreensão da real condição do verde urbano (LINDENMAIER e SOUZA, 2015).

$O$ conhecimento da interação entre a vegetação e o meio que a cerca tem grande importância para o entendimento dos fenômenos naturais, sendo o sensoriamento remoto, uma importante ferramenta para o conhecimento da cobertura vegetal em áreas urbanas (LUCHIARI, 2001). Nesse contexto, a espectrorradiometria possibilita detectar a resposta espectral de alvos específicos, podendo ser feita em laboratório, permitindo assim minimizar as interferências dos fatores ambientais presentes nas leituras de outros sensores (SILVA et al., 2012).

A definição mais clássica e difundida de sensoriamento remoto foi proposta por Elachi (1987), tratando-o como a ciência e a arte de se obter informações sobre um objeto, área ou fenômeno, por meio da análise de dados provenientes de um sistema com a ausência de contato físico. Essa tecnologia aborda a resposta de objetos expostos à radiação, sendo o solo e a vegetação os elementos mais pesquisados na área das ciências agrárias.

A radiação eletromagnética na superfície vegetal apresenta diferentes respostas morfológicas e fisiológicas no componente vegetal, ou no ecossistema como um todo. A resposta espectral de uma floresta, por exemplo, quando comparada a uma folha, apresenta maior influência do solo, das condições climáticas e das alterações da vegetação, sendo a reflectância espectral de folhas isoladas diferente daquela apresentada pelos dosséis (LILLESAND e KIEFER, 1994; SILVA e PONZONI, 1995).

A interação entre a radiação eletromagnética e a estrutura celular da folha ocorre em função da composição, morfologia e estrutura interna. Dessa forma, as características geneticamente controladas, apresentarão diferenças no comportamento espectral entre grupos geneticamente distintos (PONZONI, 2001). A quantidade de energia absorvida, transmitida ou refletida pelas folhas destaca Moreira (2011), pode diferir de uma espécie para outra, ou até mesmo, dentro da própria espécie, porque existem, dentre outros fatores, influência direta ou indireta das condições ambientais nessa interação.

No que se refere à resposta espectral de uma folha típica Knipling (1970) e Guyot et al. (1989), consideram três faixas do espectro eletromagnético: a região do visível $(0,4-$ $0,7 \mu \mathrm{m})$; do infravermelho próximo $(0,7-1,3$ $\mu \mathrm{m})$ e do infravermelho médio $(1,3-2,6 \mu \mathrm{m})$. Além disso, o comportamento espectral das folhas também pode ser caracterizado pela banda "red edge" no intervalo de comprimento de onda entre $0,68-0,75 \mu \mathrm{m}$. O ponto de inflexão com elevada declividade representa a transição abrupta entre a forte absorção nos comprimentos de ondas do visível, principalmente no vermelho, e a reflectância no infravermelho próximo (CURRAN et al., 1991; FILELLA e PEÑUELAS, 1994; MILLER et al., 1990).

Diversos índices de vegetação têm sido propostos na literatura com o objetivo de explorar as propriedades espectrais da vegetação, especialmente nas regiões do visível e do infravermelho próximo (PONZONI, 2012). Amplamente usados como indicadores da presença e condição de vegetação, esses índices podem ser obtidos tanto de dados coletados por satélites quanto por equipamentos próximos ao objeto de estudo, como é o caso dos espectrorradiômetros (MOREIRA, 2011). Os índices de vegetação resultam de combinações lineares, razões ou transformações ortogonais de bandas espectrais, realçando o sinal da vegetação, que minimizam as variações na irradiância solar e os efeitos do substrato do dossel vegetal (JACKSON e HUETE, 1991). Além disso, possibilitam comparações espaciais e temporais da atividade fotossintética terrestre, facilitando, assim, o monitoramento sazonal, interanual e variações de longo prazo dos parâmetros estruturais, fisiológicos e biofísicos da vegetação (WANG et al., 2003). 
O sensor orbital WordView-2, lançado em 2009, consiste no primeiro sistema de alta resolução espacial que possui oito bandas (RIBEIRO; FONSECA e KUX, 2011). Isso contribuiu para uma melhor visão dos ambientes intra-urbanos (PASSO et al., 2013), sendo que, o diferencial em uma imagem de satélite está no tipo de sensor usado (PARANHOS FILHO et al., 2008). Diante do exposto, a pesquisa foi desenvolvida com o objetivo de caracterizar e comparar o comportamento espectral de seis espécies arbóreas distintas, por meio de valores médios de reflectâncias foliares obtidas em laboratório, identificando os índices de vegetação e as bandas espectrais do satélite WorldView-2 mais adequadas na distinção das espécies.

\section{Material e métodos}

O trabalho foi desenvolvido no município de Santa Maria, no estado do Rio Grande do Sul, na região da depressão central, cuja coordenada corresponde à latitude $29^{\circ} 43^{\prime} 23^{\prime}$ ' S e longitude 5343'15" W e altitude de $95 \mathrm{~m}$ (SILVA et al., 2007). O clima da região, de acordo com a classificação de Köppen, é do tipo subtropical úmido "Cfa-com verão quente", temperaturas máximas de $23-26^{\circ} \mathrm{C}$ e mínimas de $11-14{ }^{\circ} \mathrm{C}$, e precipitação variando de 1.600 a $1.900 \mathrm{~mm}$ (ALVARES et al., 2013; ALVARES et al., 2014).

$\mathrm{O}$ estudo abrangeu áreas verdes situadas em torno da Universidade Federal de Santa Maria (UFSM), onde foram coletadas e identificadas seis espécies florestais: Eucalyptus sp., Ficus benjamina L., Inga marginata Mart., Schinus terebinthifolius Raddi, Psidium cattleyanum Sabine, Handroanthus chrysotrichus (Mart. Ex A. DC.) Mattos, utilizadas na arborização do Campus universitário. A coleta das folhas ocorreu no dia 2 de setembro de 2015 , entre às $7 \mathrm{~h}$ e $8 \mathrm{~h}$, nesse momento, a temperatura variava de 8,7 a $10,5^{\circ} \mathrm{C}$ e a umidade do ar entre 87 e 94 $\%$, sem ocorrência de chuva (INMET, 2015).
O comportamento espectral (reflectância) de cada espécie foi registrado por meio do espectrorradiômetro FieldSpec $₫ 3$, modelo RST $3 Z C$, conectado à esfera integradora. As leituras foram realizadas entre 1-2 horas após a coleta das folhas (sete por espécie).

As folhas foram posicionadas com a face adaxial para o interior da esfera, sendo tomadas 10 leituras amostrais em cada uma delas. Os dados obtidos desse procedimento constituíram uma matriz com valores médios por espécie, cujos comprimentos de ondas compreendem o intervalo entre 350 e $2.500 \mathrm{~nm}$. Os dados originais foram convertidos para o formato texto “.txt" e determinada a média da reflectância das sete leituras de cada espécie.

As médias das reflectâncias possibilitaram calcular os valores de 61 índices de vegetação, conforme listados na Tabela 1, extraídos de Li et al. (2010).

Após processamento dos índices foi construída uma matriz de índice x espécie. Com os dados dessa matriz foi aplicada a técnica multivariada da análise de componentes principais, visando diminuir o conjunto de dados eliminando os componentes que tiveram pouca influência na variância total. A seleção dos índices de vegetação foi realizada com base nos componentes mais representativos, a partir dos valores decrescentes de desvio padrão por índice. O comportamento das espécies ao longo do espectro eletromagnético foi realizado com base nas bandas espectrais do satélite WorldView-2 (Tabela 2).

As diferenças entre os comportamentos espectrais das espécies por bandas do sensor foram testadas por meio de análise de variância. Quando detectado diferença em algum par de médias foi aplicado o teste de comparações múltiplas Tukey HSD (honest signifi cant differences) para verificar onde ocorreu (ram) a (s) diferença (s). As análises estatísticas foram realizadas com auxílio do programa Microsoft Office Excel e no software R. 
Tabela 1 - Funções de índices de vegetação utilizados para avaliar a reflectância de seis espécies presentes na arborização urbana da Universidade Federal de Santa Maria, RS.

Table 1 - Definition of vegetation indices used for reflectance evaluation for six species in urban afforestation at the Federal University of Santa Maria, RS.

\begin{tabular}{|c|c|c|c|c|c|}
\hline Nome & Descrição & $\lambda$ & Nome & Descrição & $\lambda$ \\
\hline $1 . G$ & Green & $554-677$ & 32.GM & Modelo Green (G-M) & $750-550$ \\
\hline 2.ZTN & $\begin{array}{l}\text { Zarco-Tejada \& Muller } \\
\text { (ZTM) }\end{array}$ & $750-710$ & 33.GNDVI & Diferença normalizada no verde & $\begin{array}{l}801-550- \\
800-550\end{array}$ \\
\hline 3.PSSra & $\begin{array}{l}\text { Pigment specific simple } \\
\text { ratio a }\end{array}$ & $800-600$ & 34.PSNDb & $\begin{array}{c}\text { Diferença normalizada pigmento } \\
\text { especifico b }\end{array}$ & $800-635$ \\
\hline 4.PSSrb & $\begin{array}{l}\text { Pigment specific simple } \\
\text { ratio } b\end{array}$ & $800-635$ & 35.PSNDc & $\begin{array}{c}\text { Diferença normalizada pigmento } \\
\text { especifico c }\end{array}$ & $800-470$ \\
\hline 5.PSSrc & $\begin{array}{l}\text { Pigment specific simple } \\
\text { ratio c }\end{array}$ & $800-470$ & 36.NPCI & $\begin{array}{c}\text { Razão Normalizada pigmentos } \\
\text { clorofila }\end{array}$ & $680-430$ \\
\hline 6.SRPI & $\begin{array}{l}\text { Simple ratio pigment } \\
\text { index }\end{array}$ & $430-680$ & 37.NPQI & Normalizado Phaeophytinization & $415-435$ \\
\hline 7.VIopt2 & $\begin{array}{l}\text { Optimized vegetation } \\
\text { index } 2\end{array}$ & $760-730$ & 38.PRI & Reflectância fotoquímica & $\begin{array}{l}531-570- \\
530-570\end{array}$ \\
\hline 8.SR1 & Simple ratio 1 & $810-670$ & 39.SIPI & Estrutura de pigmento intensivo & $\begin{array}{l}800-445- \\
400-680\end{array}$ \\
\hline 9.SR2 & Simple ratio 2 & $800-550$ & 40.ND705 & $\begin{array}{l}\text { Vegetação da Diferença } \\
\text { Normalizada } 705\end{array}$ & $750-705$ \\
\hline 10.SR3 & Simple ratio 3 & $700-670$ & 41.mND705 & $\begin{array}{l}\text { Vegetação da Diferença } \\
\text { Normalizada } 705 \text { Modificado }\end{array}$ & $750-705-445$ \\
\hline 11.SR4 & Simple ratio 4 & $740-720$ & 42.mSR705 & Razão simples modificado & $750-445-705$ \\
\hline 12.SR5 & Simple ratio 5 & $675-700-650$ & 43.PSRI & Reflectância de plantas senescentes & $680-500-750$ \\
\hline 13.SR6 & Simple ratio 6 & $675-700$ & 44.RGR & Razão Vermelho - Verde & $\begin{array}{l}612-660- \\
510-560\end{array}$ \\
\hline 14.SR7 & Simple ratio 7 & $672-550-708$ & 45.NDI1 & Índice de Diferença Normalizada 1 & $780-710-680$ \\
\hline 15.SR8 & Simple ratio 8 & $860-550-708$ & 46.NDI2 & Índice de Diferença Normalizada 2 & $850-710-680$ \\
\hline 16.SR9 & Simple ratio 9 & $750-700$ & 47.NDVI1 & NDVI & $\begin{array}{l}734-747- \\
715-726\end{array}$ \\
\hline 17.SR10 & Simple ratio 10 & $750-550$ & 48.NDVI2 & NDVI & $800-700$ \\
\hline 18.SR11 & Simple ratio 11 & $900-680$ & 49.NDVI3 & NDVI & $780-550$ \\
\hline 19.SR12 & Simple ratio 12 & $550-850$ & 50.NDVI4 & NDVI & $800-600$ \\
\hline 20.SR13 & Simple ratio 13 & $710-850$ & 51.NDVI5 & NDVI & $900-680$ \\
\hline 21.SR14 & Simple ratio 14 & $415-695$ & 52.NDVI6 & NDVI & $760-708$ \\
\hline 22.SR15 & Simple ratio 15 & $415-710$ & 53.NDVI7 & NDVI & $573-440$ \\
\hline 23.SR16 & Simple ratio 16 & $750-705$ & 54.NDVI8 & NDVI & $503-483$ \\
\hline 24.SR17 & Simple ratio 17 & $810-560$ & 55.NDVI9 & Índice de reflectância modificado & $700-670-550$ \\
\hline 25.SR18 & Simpre ratio 18 & $787-765$ & & & \\
\hline 26.DI1 & Diferença 1 & $800-550$ & 56.NDVI10 & Índice CARI transformado & $700-670-550$ \\
\hline 27.DVI & Vegetação Diferenciada & $800-680$ & 57.OSAVI & $\begin{array}{l}\text { Índice de vegetação ótimo ajustado } \\
\text { para solo }\end{array}$ & $800-670$ \\
\hline 28.VIopt1 & Vegetação Otimizada & $760-730$ & 58.REIP & Índice ponto de inflexão RedEdge & $\begin{array}{l}670-780- \\
700-740\end{array}$ \\
\hline 29.DD & Diferença dupla & $\begin{array}{l}750-720- \\
700-670\end{array}$ & 59.TVI & Índice de vegetação transformado & $750-550-670$ \\
\hline 30.RM & $\begin{array}{l}\text { Modelo Red-Edge (R- } \\
\text { M) }\end{array}$ & $750-720$ & 60.LAIVI & Razão entre TCARI e OSAVI & $\begin{array}{l}\text { TCARI/ } \\
\text { OSAVI }\end{array}$ \\
\hline 31.MTCI & $\begin{array}{l}\text { Índice terrestre de } \\
\text { clorofila (MTCI) }\end{array}$ & $\begin{array}{l}750-710- \\
710-680\end{array}$ & 61.RDVI & $\begin{array}{l}\text { Vegetação da diferença Re- } \\
\text { normalizada }\end{array}$ & $800-670$ \\
\hline
\end{tabular}


Tabela 2 - Comprimentos de onda por bandas espectrais do satélite WorldView-2.

Table 2 -WorldView-2 satellite spectral bands wavelengths.

\begin{tabular}{lccc}
\hline & Banda espectral & Nomenclatura utilizada & Comprimento de Onda (nm) \\
\hline 1 & Costal & MS1 & $400-450$ \\
2 & Azul & MS2 & $450-510$ \\
3 & Verde & MS3 & $510-580$ \\
4 & Amarelo & MS4 & $585-625$ \\
5 & Vermelho & MS5 & $630-690$ \\
6 & RedEdge & RedEdge & $705-745$ \\
7 & Infravermelho Próximo 1 & NIR1 & $770-895$ \\
8 & Infravermelho Próximo 2 & NIR2 & $860-1040$
\end{tabular}

Fonte: Adaptado de Padwick et al. (2010).

Os mesmos dados utilizados nos testes de comparações múltiplas serviram de base para a análise de agrupamento. Foi empregado o método Ward de agrupamento e a distância Euclidiana como medida de dissimilaridade, sendo esta análise realizada com auxílio do programa PC-ORD 6.0 (MCCUNE E MEFFORD, 2011).

Para obtenção e processamento dos dados espectrais foram utilizados os programas ASD ViewSpecPro 4.05 e SAMS 3.2. A partir dos valores médios de reflectância das espécies na região do visível e do infravermelho próximo, elaborou-se gráficos.

\section{Resultados e Discussão}

A análise de componentes principais da matriz de dados dos índices de vegetação apontou o primeiro componente como responsável por $90,94 \%$ de toda a variância dos dados. Com apenas um eixo foi possível explicar a relação entre os índices e as espécies arbóreas estudadas (Figura 1).

Os índices de vegetação que mais contribuíram com variância dos dados explicada na análise de componentes principais foram
VIOPT1 (Índice de Vegetação Otimizada) e SR8 (Razão Simples 8). De acordo com Schroder et al. (2015), os índices de vegetação, embora em pequena escala, podem ser influenciados pela estação do ano e pela orientação e posição na copa. A dinâmica sazonal de clorofila, comentam Goergen et al. (2015), também pode exercer influência, por exemplo, o Eucalyptus grandis apresentou maiores teores no período verão-outono, acarretando em discrepância nos valores dos índices nesse período.

Georgen et al. (2016), com base em imagens TM/Landsat 5, relataram que ao avaliar as respostas espectrais de plantios de Eucalyptus dunnii e Eucalyptus urograndis, os índices de vegetação "Razão Simples-0,5”, "Razão Simples-0,25" e "Green Índice de Vegetação da Diferença Normalizada-GNDVI" foram eficazes na distinção dessas espécies dentre todas as situações avaliadas.

As condições de cultivo e a sua influência nos índices de vegetação também são reportadas por Motomiya et al. (2009). Na cultura do algodoeiro com utilização de sensor óptico, o índice de razão simples e índice de clorofila são decrescentes em condições de cultivo com menor disponibilidade de nitrogênio. 


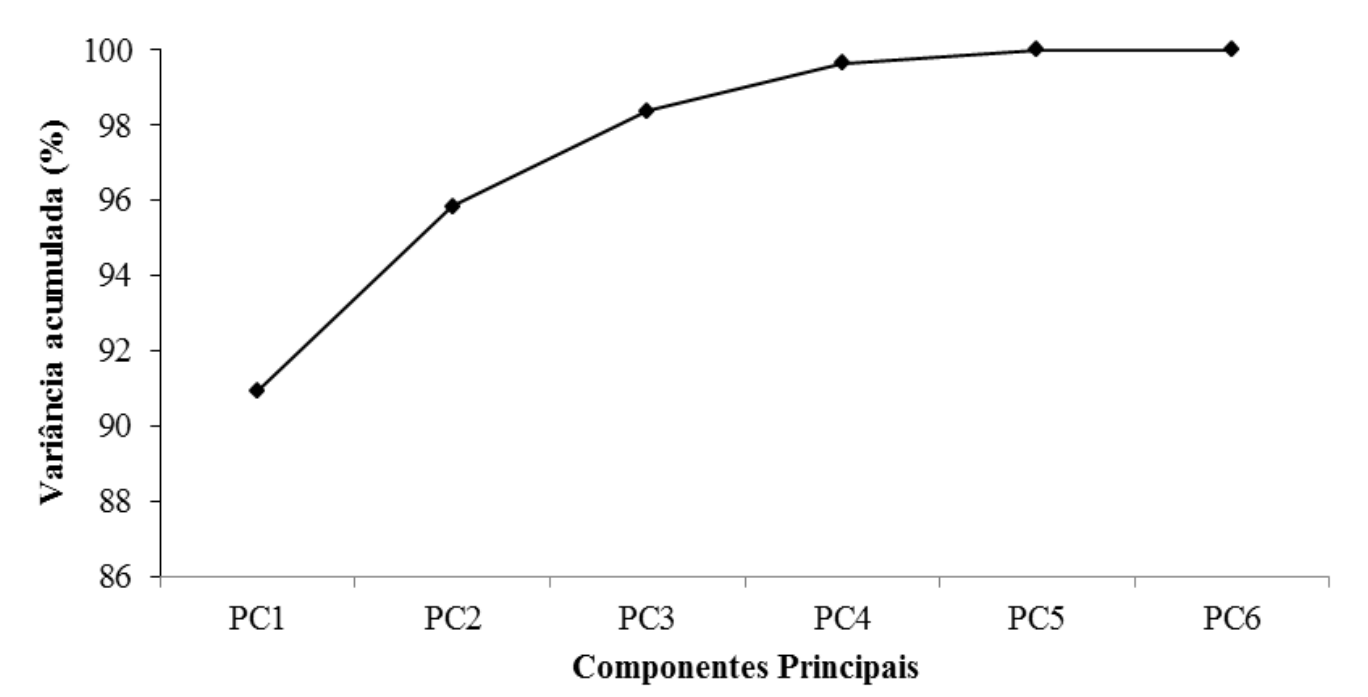

Figura 1 - Análise de componentes principais com base na matriz de índices de vegetação em função de seis espécies arbóreas. Figure 1 - Principal component analysis based on the vegetation indices matrix for six tree species.

Li et al. (2010), ao pesquisarem variações nos estágios de crescimento em culturas agrícolas, tais como milho (Zea mays L.) e algodão (Gossypium hirsutum L.), encontraram o melhor desempenho para os índices VIOPT1 e VIOPT2, que explicaram 58-59\% da variação dos dados. Isto porque esses índices abrangem mais faixas do espectro eletromagnético, usando a banda RedEdge juntamente com bandas verdes, vermelhas ou NIR, sendo estes comprimentos de onda mais sensíveis as variações químicas e estruturais da folha.

As análises de variâncias das médias de reflectâncias demonstraram que, pelo menos, um par de espécies em todas as bandas espectrais do Sensor Worldview 2 apresentaram diferenças entre si, pelo teste Tukey HSD (Tabela 3).

Vários fatores têm sido mencionados na literatura como determinantes na resposta espectral de uma espécie. Gausman (1984), analisando a reflectância da folha de laranjeira, Citrus sinensis (L.) Osbeck., em diferentes comprimentos de onda do visível $(450,550$ e $650 \mathrm{~nm})$, concluiu que a concentração de clorofila, volume de ar do mesófilo, quantidade de água e espessura foram diferentes entre as folhas de sombra e sol, resultando em respostas espectrais distintas. Carte e Knapp (2001) apontaram que diferenças espectrais em uma mesma espécie podem ocorrer quando se compara folhas com e sem estresse hídrico. Os mesmos autores mencionam que a resposta ao estresse na faixa do vermelho é atribuída à tendência das folhas estressadas perderem clorofila, diminuindo consideravelmente a absortância.

Silva et al. (2012), analisando diferentes posições de coleta de folhas (norte, sul, leste e oeste) na parte inferior da copa de Eucalyptus grandis W. Mill ex Maiden, concluíram que a reflectância diferiu com a posição de coleta das folhas, devido à orientação solar. Brandelero et al. (2012), avaliando as leituras de reflectância em folhas de árvores de bordadura e de interior em um povoamento jovem de E. grandis, considerando as folhas das partes inferior, média e superior das copas, nas regiões do visível (400 a $690 \mathrm{~nm}$ ) e do infravermelho próximo (650 a $890 \mathrm{~nm}$ ), afirmaram que as árvores do interior (folha mediana) e bordadura (folha superior) compõem as classes indicadoras para o estudo desta espécie. 
Tabela 3 - Análise de variâncias da reflectância média de seis espécies arbóreas por banda espectral do Sensor Worldview 2. Table 3 - Analysis of variance for the mean average reflectance by each spectral band of the Worldview 2 satellite sensor for six tree species.

\begin{tabular}{|c|c|c|c|c|c|c|}
\hline \multirow{4}{*}{ MS1 } & F.V. & GL & SQ & $\mathbf{Q M}$ & $\mathbf{F}$ & $\operatorname{Pr}(>\mathbf{F})$ \\
\hline & Dimensão & 5 & 0.04767 & 0.009534 & 35.37 & $<2 \mathrm{e}-16 * * *$ \\
\hline & Resíduo & 300 & 0.08087 & 0.000270 & & \\
\hline & Total & 305 & 0.12854 & 0.009804 & & \\
\hline \multirow{4}{*}{ MS2 } & F.V. & GL & SQ & $\mathbf{Q M}$ & $\mathbf{F}$ & $\operatorname{Pr}(>\mathbf{F})$ \\
\hline & Dimensão & 5 & 0.05440 & 0.01088 & 269.5 & $<2 \mathrm{e}-16^{* * *}$ \\
\hline & Resíduo & 360 & 0.01453 & 0.00004 & & \\
\hline & Total & 365 & 0.06893 & 0.01092 & & \\
\hline \multirow{4}{*}{ MS3 } & F.V. & GL & SQ & QM & $\mathbf{F}$ & $\operatorname{Pr}(>\mathbf{F})$ \\
\hline & Dimensão & 5 & 0.1136 & 0.022716 & 73.01 & $<2 \mathrm{e}-16^{* * *}$ \\
\hline & Resíduo & 420 & 0.1307 & 0.000311 & & \\
\hline & Total & 425 & 0.2443 & 0.023027 & & \\
\hline \multirow{4}{*}{ MS4 } & F.V. & GL & SQ & QM & $\mathbf{F}$ & $\operatorname{Pr}(>\mathbf{F})$ \\
\hline & Dimensão & 5 & 0.04373 & 0.008747 & 222.5 & $<2 \mathrm{e}-16^{* * * *}$ \\
\hline & Resíduo & 240 & 0.00943 & 0.000039 & & \\
\hline & Total & 245 & 0.05316 & 0.008786 & & \\
\hline \multirow{4}{*}{ MS5 } & F.V. & GL & SQ & QM & $\mathbf{F}$ & $\operatorname{Pr}(>\mathbf{F})$ \\
\hline & Dimensão & 5 & 0.05328 & 0.010656 & 343.2 & $<2 \mathrm{e}-16^{* * *}$ \\
\hline & Resíduo & 360 & 0.01118 & 0.000031 & & \\
\hline & Total & 365 & 0.06446 & 0.010687 & & \\
\hline \multirow{4}{*}{ RED EDGE } & F.V. & GL & SQ & QM & $\mathbf{F}$ & $\operatorname{Pr}(>\mathbf{F})$ \\
\hline & Dimensão & 5 & 0.1499 & 0.02999 & 2.847 & $0.0162 *$ \\
\hline & Resíduo & 240 & 2.5277 & 0.01053 & & \\
\hline & Total & 245 & 2.6776 & 0.04052 & & \\
\hline \multirow{4}{*}{ NIR1 } & F.V. & GL & SQ & QM & $\mathbf{F}$ & $\operatorname{Pr}(>\mathbf{F})$ \\
\hline & Dimensão & 5 & 1.0316 & 0.20632 & 21770 & $<2 \mathrm{e}-16^{* * * *}$ \\
\hline & Resíduo & 750 & 0.0071 & 0.00001 & & \\
\hline & Total & 755 & 1.0387 & 0.20633 & & \\
\hline \multirow{4}{*}{ NIR2 } & F.V. & GL & SQ & QM & $\mathbf{F}$ & $\operatorname{Pr}(>\mathbf{F})$ \\
\hline & Dimensão & 5 & 1.4790 & 0.29579 & 4909 & $<2 \mathrm{e}-16^{* * *}$ \\
\hline & Resíduo & 1080 & 0.0651 & 0.00006 & & \\
\hline & Total & 1085 & 1.5441 & 0.29585 & & \\
\hline
\end{tabular}

Legenda: $\mathrm{GL}=$ graus de liberdade; $\mathrm{SQ}=$ soma dos quadrados; $\mathrm{QM}=$ Quadrado médio; $\mathrm{F}=$ razão entre o modelo e o seu erro; $\mathrm{Pr}=$ probabilidade; *= existe diferença significativa; $* * *=$ existe diferença altamente significativa.

Caption: $\mathrm{GL}=$ degrees of freedom; $\mathrm{SQ}=$ sum of squares; $\mathrm{QM}=$ mean square; $\mathrm{F}=$ ratio between Mean Square Model and Mean Square Error; $\mathrm{PR}=$ probability; $*$ = significant difference; $=$ highly significant difference.

No presente estudo foi verificado que as curvas de reflectância apresentam comportamento direcional-hemisférica. Segundo Ponzoni (2012), esta condição apresenta relação com a região do visível (domina a reflectância), com região do infravermelho próximo (pequena absorção da radiação solar e considerável espalhamento no interior da folha) e a região do infravermelho médio (efeito da água líquida na reflectância).

As análises de agrupamento corroboraram quase em sua totalidade com os resultados das comparações múltiplas das médias (Figura 2). O reconhecimento de padrões se dá pelas propriedades espectrais distintas nas diferentes espécies, que as agruparam e distinguiram por bandas. Esta técnica tem sido aplicada com sucesso em estudo de espectrorradiometria de espécies vegetais (PAZ et al., 2009; WATANABE et al., 2011).

A banda que apresentou a menor relação entre o agrupamento e as diferenças das médias por espécie foi a MS5. Neste caso, a interpretação foi realizada unicamente com os resultados do teste Tukey HSD. As bandas MS1, MS2, MS3, MS4 e MS5 obtiveram um comportamento semelhante, todavia, foram diferenciadas nestas bandas as espécies $F$. benjamina (MS1, MS2, 
MS4 e MS5), I. marginata (MS3 e MS4), e $P$. cattleyanum (MS3). Na banda RedEdge não foi possível fazer a separação de espécies, posto que, das 15 comparações realizadas, 13 foram não significativas.

As diferenças de reflectâncias das seis espécies foram mais evidentes nas bandas NIR1
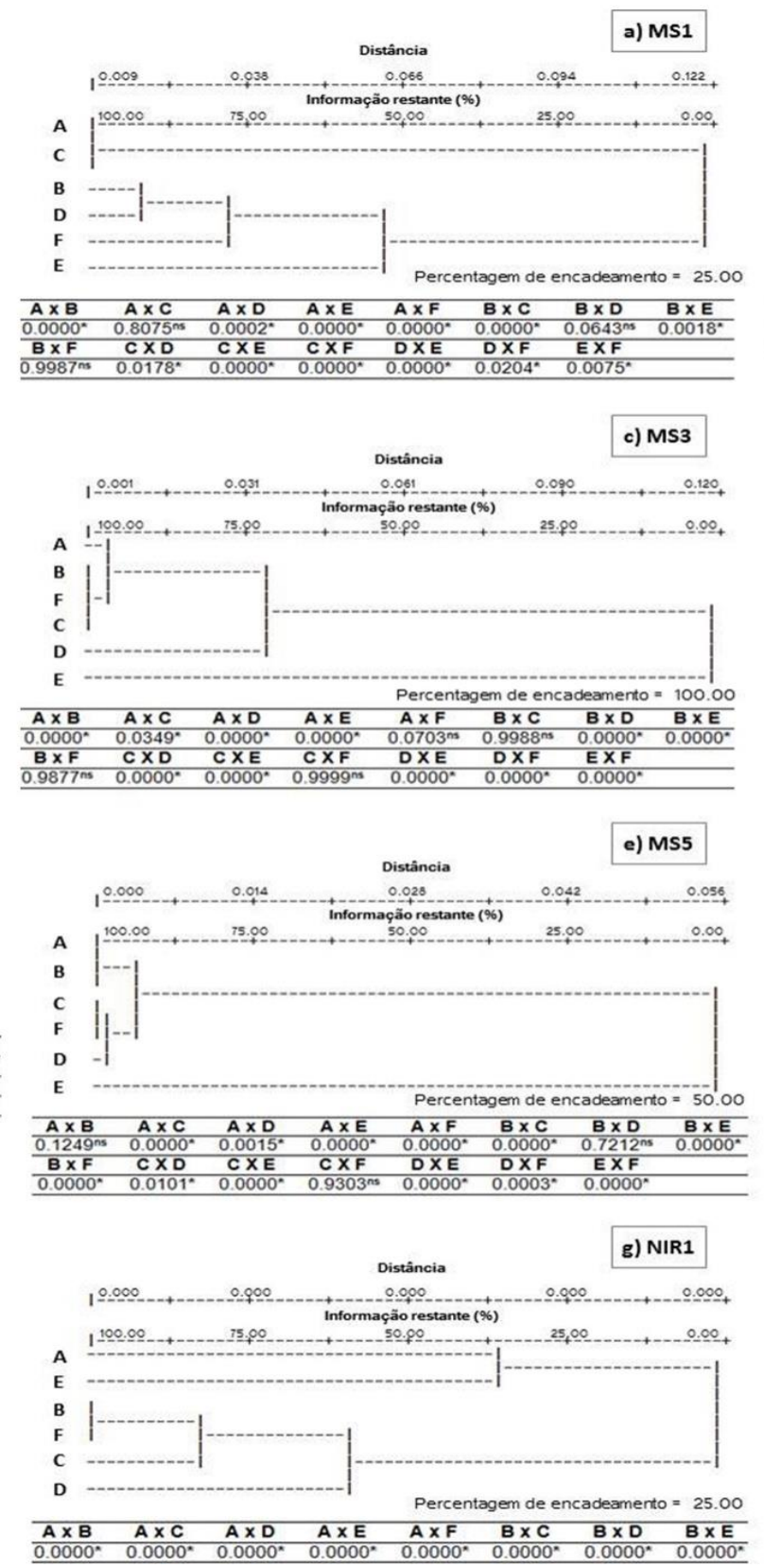

(Infravermelho Próximo 1) e NIR2 (Infravermelho Próximo 2), em que todas apresentaram comportamento espectral distinto (Figura 2). Dessa forma, é possível inferir que estas são as melhores bandas do sensor Worldview 2 para distinguir as espécies investigadas.
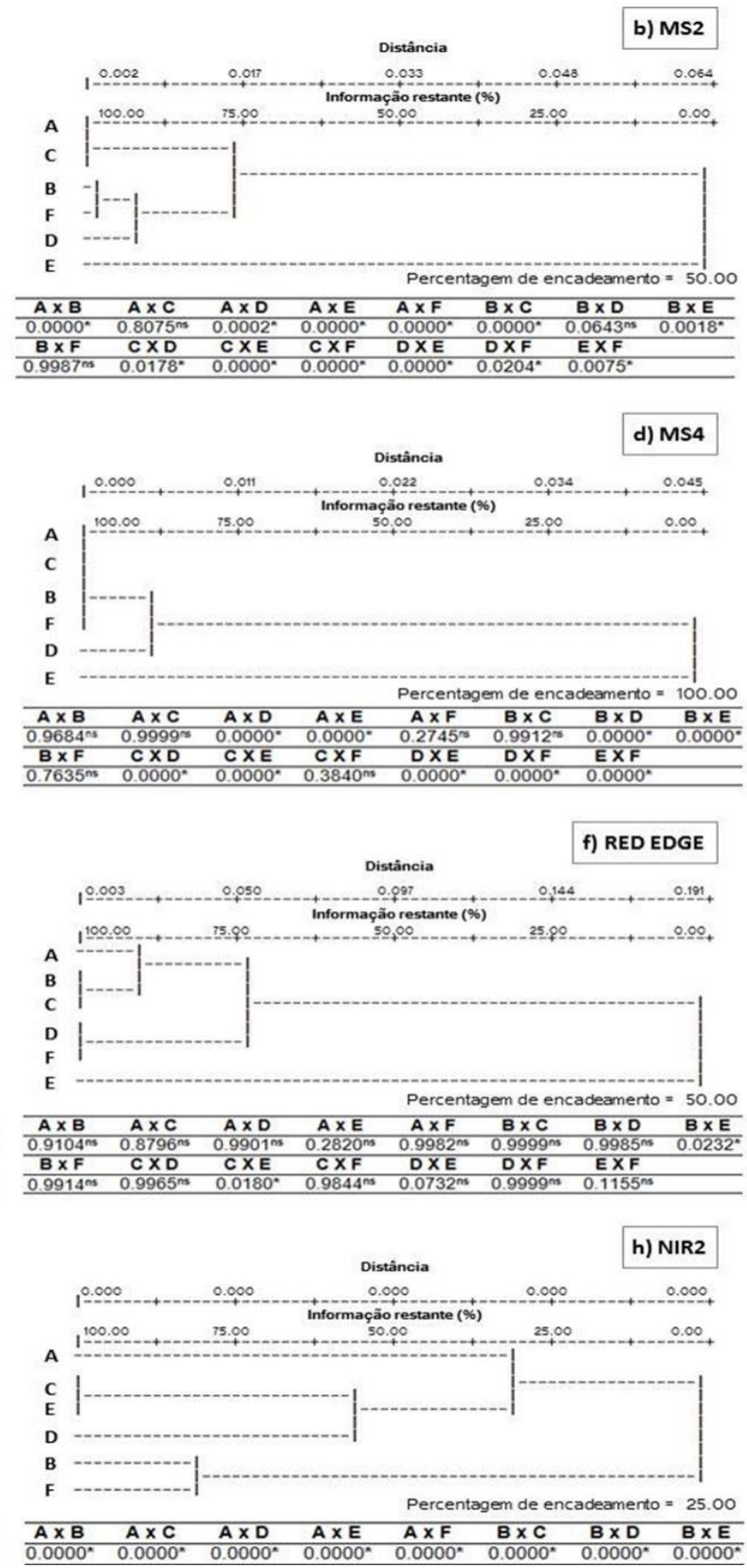

Figura 2 - Análise da reflectância de seis espécies arbóreas por banda espectral do Sensor Worldview 2 por meio do Cluster hierárquico e do teste TUKEY HSD. Espécies: A - Eucalyptus sp.; B - Schinus terebinthifolius; C - Handroanthus chrysotrichus; D - Inga marginata $; \mathrm{E}$ - Ficus benjamina $; \mathrm{F}$ - Psidium cattleyanum; * = diferença significativa entre médias; ns $=$ diferença não significativa entre médias.

Figure 2 - Reflectance analysis through the hierarchical clustering analysis and TUKEY HSD test by each spectral band of the Worldview 2 satellite sensor for six tree species. Species: A - Eucalyptus sp.; B - Schinus terebinthifolius; C Handroanthus chrysotrichus; D - Inga marginata; E - Ficus benjamina; F - Psidium cattleyanum; * = significant difference between averages; NS = no significant difference between averages. 
Os comportamentos espectrais das espécies estudadas possuem formatos similares, com algumas diferenças nos valores das amostras. Todas as espécies apresentaram um padrão similar de uma curva espectral típica de folha verde (Figura 3). Situação semelhante foi observado por Silva et al. (2012), ao estudar o comportamento espectral de E. grandis através de folhas que ainda realizavam fotossíntese, essas apresentaram bandas de absorção nas regiões do azul e vermelho (450 nm e $650 \mathrm{~nm})$.

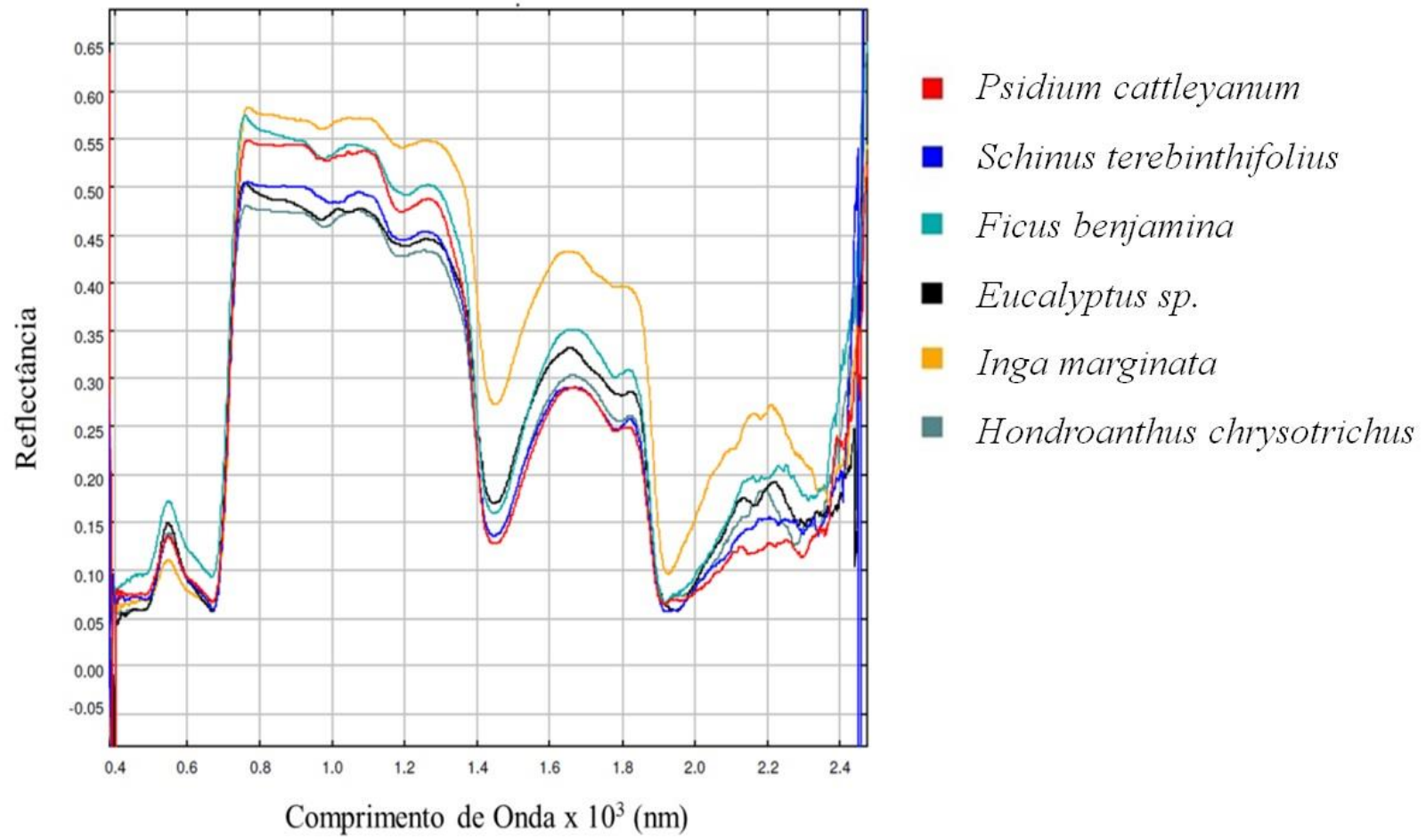

Figura 3 - Assinatura espectral média de seis espécies arbóreas coletadas no Campus da Universidade Federal de Santa Maria, RS, obtidas por meio do espectrorradiômetro FieldSpec®3, modelo RST 3ZC.

Figure 3 - Average spectral - response curves for six tree species collected on the Campus of the Federal University of Santa Maria, RS, obtained through the FieldSpec3 Spectroradiometer, RST 3ZC model.

Deve-se considerar em análises espectrais que as propriedades óticas das folhas podem ser afetadas por fatores como idade, deficiência de nutrientes e doenças. Durante o seu desenvolvimento, comentam Rangel et al. (2001), tem-se o rápido acréscimo percentual de clorofila nos estágios iniciais, com redução nos estágios subsequentes, até atingir a coloração verde característica de uma espécie.

Nas bandas que compreendem a região do visível (400 a 700 nm) Ficus benjamina e Inga marginata apresentaram os maiores e menores valores de reflectância, respectivamente. Nessas, o comportamento foi mais acentuado entre 520 nm e $600 \mathrm{~nm}$, referente à região espectral do verde. As demais espécies apresentaram curvas de respostas espectrais semelhantes (Figura 4a). Nas bandas do visível, a resposta espectral é explicada devido às diferenças nos componentes químicos presente nas plantas, incluindo a quantidade de pigmentos fotossintéticos (PONZONI, 2001).

$\mathrm{Na}$ região do visível, segundo Ponzoni e Shimabukuro (2007), as folhas têm baixa reflectância (menos de 10\%), explicada pela correlação entre os pigmentos existentes e a absorção de energia. Cada pigmento tem uma afinidade peculiar com diferentes faixas 
espectrais. O principal deles, responsável pela absortância, é a clorofila, que absorve REM na região do vermelho $(670 \mathrm{~nm})$. Os pigmentos acessórios, como os carotenos e xantofilas, predominantemente absorvem radiação na região azul (próximo a $445 \mathrm{~nm}$ ).

Para melhor diferenciar as espécies em estudos futuros, recomenda-se incluir uma análise da quantidade dos pigmentos de cada uma delas, visto que, conforme mencionado por Shimabukuro e Ponzoni (2012), os diferenciados pigmentos presente nas folhas resultam nas diferenças de reflectância em cada espécie.

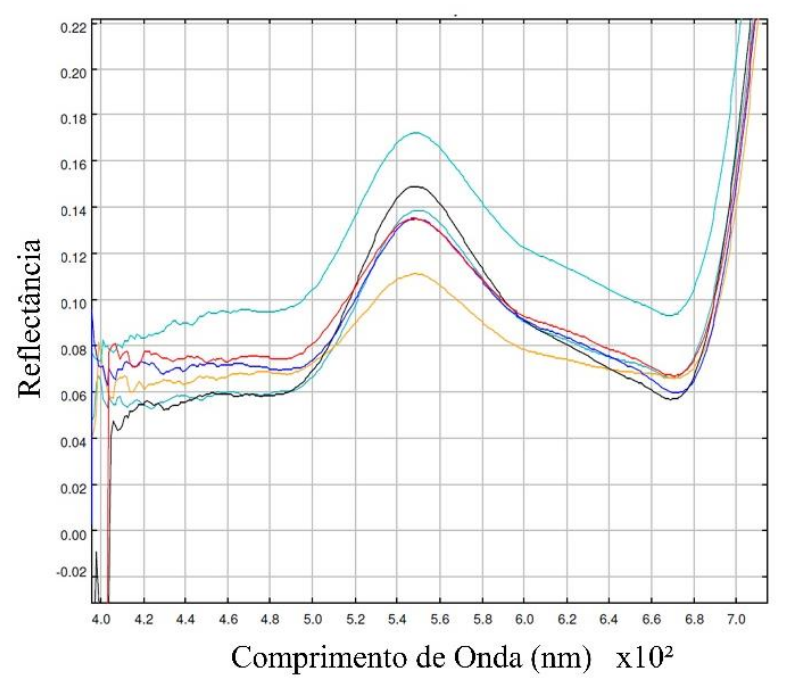

a) Faixa do Visível

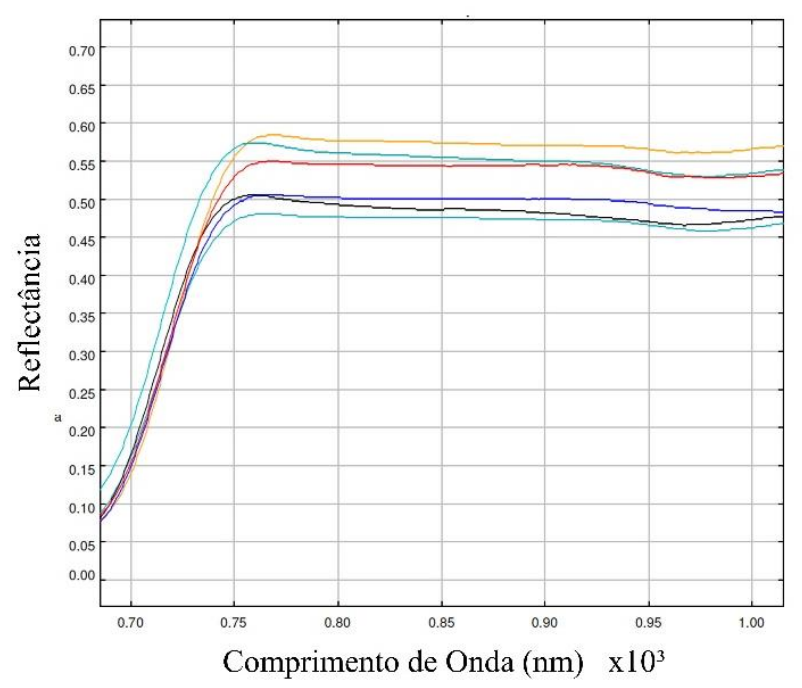

b) Faixa do Infravermelho Próximo
Psidium cattleyanum

Schinus terebinthifolius
Ficus benjamina

Eucalyptus sp.
Inga marginata

Hondroanthus chrysotrichus

Figura 4 - Assinatura espectral de seis espécies arbóreas coletadas no Campus da Universidade Federal de Santa Maria, RS, obtidas por meio do espectrorradiômetro FieldSpec®3, modelo RST 3ZC. a) Faixa do visível (400 a 700 nm); b) Faixa do infravermelho próximo (700 a $1300 \mathrm{~nm})$.

Figure 4 - Average spectral - response curves for six tree species collected on the Campus of the Federal University of Santa Maria, RS, obtained through the FieldSpec3 Spectroradiometer, RST 3ZC model. a) Visible region (400 a $700 \mathrm{~nm}$ ); b) Near infrared region (700 a $1300 \mathrm{~nm})$.

Mudanças no teor de clorofila tendem a produzir grandes diferenças na reflectância e na transmitância das folhas, o que por sua vez pode ajudar na discriminação dos tipos de vegetação (GITELSON, 2005). Dessa maneira, os distintos valores de reflectância observados nas seis espécies arbóreas, certamente, estão associados aos teores de clorofila de cada uma delas.

Gitelson e Merzlyak (1996) verificaram que mesmo um pequeno aumento no conteúdo de clorofila em folhas verdes leva a uma diminuição significativa na reflectância, que podem estar associadas ao conteúdo de clorofila das folhas.

$\mathrm{Na}$ faixa espectral do infravermelho próximo (NIR), que vai de $700 \mathrm{~nm}$ a $1.300 \mathrm{~nm}$, os arranjos celulares peculiares de cada espécie afetam, diretamente, a sua reflectância, gerando diferenças pouco expressivas (Figura 4b). Essa faixa do espectro eletromagnético não é adequada para diferenciar as espécies investigadas, visto que, elas não apresentaram diferenças expressivas em suas curvas.

A resposta espectral de cinco espécies arbustivas foi caracterizada por Cardoso e Ponzoni (1996). Os autores não encontraram 
diferenças significativas na região do visível, contudo, diferenças acentuadas foram registradas na região do NIR, devido à influência da estrutura do mesófilo. A espécie Tibouchina fothergillae (DC.) Cogn, possui o parênquima paliçádico compacto, porém suas epidermes apresentarem três camadas de células cujas formas eram arredondadas e com a ocorrência de espaços intercelulares ocupados por ar. Este tecido pode ter sido o responsável por maior espalhamento da REM na região do infravermelho próximo.

Em estudo com árvores de E. grandis, Brandelero et al. (2012), não encontraram diferenças significativas nas curvas espectrais da região do infravermelho próximo entre árvores pertencentes à bordadura e exemplares no interior do povoamento, enquanto que na região do visível, as curvas foram distintas.

$\mathrm{Na}$ região do infravermelho próximo existe uma absorção pequena da radiação eletromagnética (REM) e considerável espalhamento interno na folha. A absorção da água é geralmente baixa e a reflectância espectral é quase constante nessa região. Gates et al. (1965) determinaram que a reflectância espectral de folhas no NIR é o resultado da interação da energia incidente com a estrutura do mesófilo. Fatores externos à folha, como disponibilidade de água, por exemplo, podem causar alterações na relação água-ar no mesófilo, podendo alterar a reflectância de uma folha nesta região. De maneira geral, quanto mais lacunosa for a estrutura interna foliar, maior será o espalhamento interno da radiação incidente, e consequentemente, maior será também a reflectância.

De acordo com Guyot et al. (1989), a variação do valor da reflectância, na região espectral do infravermelho próximo, é proporcional ao número de camadas e tamanho das células, bem como a orientação das paredes da folha. Desta forma, a absortância da folha nesta região espectral é baixa, e a reflectância está relacionada com a sua estrutura interna. Além disso, conforme Ponzoni e Disperati (1995), a reflectância espectral na referida região apresenta-se de forma quase constante.
No infravermelho médio (1.300 a $2.500 \mathrm{~nm}$ ), as propriedades óticas das folhas são afetadas principalmente pelo seu conteúdo de água. Após $1.300 \mathrm{~nm}$ ocorrem fortes bandas de absorção pela água $(1.450,1.950$ e $2.500 \mathrm{~nm})$, originando reflectâncias mínimas na folha (RANGEL et al., 2001).

\section{Conclusões}

Os Índices de Vegetação Otimizada (VIOPT1) e o de Razão Simples (SR8) são os mais indicados nos estudos das seis espécies arbóreas.

A espécie Ficus benjamina pode ser identificada nas bandas MS1, MS2, MS4 e MS5, enquanto que o Inga marginata se identifica nas bandas MS3 e MS4 e o Pisidium cattleyanum na banda MS3. As espécies Schinus terebinthifolius, Psidium cattleyanum e Handroanthus chrysotrichus não apresentaram diferenças expressivas em suas curvas tanto na região do visível quanto do infravermelho próximo e médio. As melhores bandas do sensor Worldview 2 para distinguir as seis espécies deste estudo foram NIR1 (Infravermelho Próximo 1) e NIR2 (Infravermelho Próximo 2).

Conforme esperado, todas as espécies seguiram o padrão espectral típico de folhas verdes, não apresentando características singulares. Apenas Inga marginata se difere ligeiramente das demais, apresentando valores mais altos de reflectância no intervalo de 750 a $2.400 \mathrm{~nm}$.

\section{Agradecimentos}

À Fundação Amazônia Paraense de Amparo à Pesquisa (FAPESPA) e à Coordenação de Aperfeiçoamento de Pessoal de Nível Superior (CAPES) pela concessão de bolsa.

\section{Referências Bibliográficas}

ALVARES, C. A.; STAPE, J. L.; SENTELHAS, 
P. C.; GONÇALVES, J. L. de M.; SPAROVEK, G. Köppen's climate classification map for Brazil. Meteorologische Zeitschrift, v. 22, n. 6, p.711-728, 2014.

ALVARES, C. A.; STAPE, J. L.; SENTELHAS, P. C.; GONÇALVES, J. L. M. Modeling monthly mean air temperature for Brazil, Theoretical and Applied Climatology, v. 113, p.407-427, 2013.

BASSO, J. M.; CORRÊA, R. S. Arborização urbana e qualificação da paisagem. Paisagem e Ambiente: Ensaios, n.34, p.129-148, 2014.

BRANDELERO, C., BERRA, E. F.; BACKES, K. S.; PEREIRA, R.; BRUN, E. J. Espectrorradiometria na região do visível e do infravermelho próximo em povoamento de Eucalyptus grandis Hill ex Maiden. Ciência Florestal, v. 22, n. 1, p. 215-222, 2012.

CARDOSO, P. C. S.; PONZONI, F. J. Caracterização espectral de folhas extraídas de cinco diferentes espécies de vegetais. In: VIII Simpósio Brasileiro de Sensoriamento Remoto, Anais. INPE, Salvador, BA, 1996. p. 891896.

CARTE, G. A.; KNAPP. Leaf optical properties in higher plants: linking spectral characteristics to stress and chlorophyll concentration. American Journal of Botany, v.88, n.4, p. 677684, 2001.

CURRAN, P. J. et al. The effect of a red leaf pigment on the relationship between red edge and chlorophyll concentration. Remote Sensing of Environment, v. 35, n. 1, p. 69-76, 1991.

ELACHI, C.; ZYL, J. V. Introduction to the physics and techniques of remote sensing. 1, ed. New York: Wiley, 1987. 551p.

FILELLA, I.; PENUELAS, J. The red edge position and shape as indicators of plant chlorophyll content, biomass and hydric status. International Journal of Remote Sensing, v. 15, n. 7, p. 1459-1470, 1994.
GATES, D.M.; KEEGAN, J.; WEIDNER, V.R. Spectral properties of plants. Applied Optics, v. 4, n. 1, p. 11-20, 1965.

GAUSMAN, H. W. Evaluation of factors causing reflectance differences between sun and shade leaves. Remote Sensing of Environment, v. 15, p.177-181, 1984.

GITELSON, A. A. Remote estimation of canopy chlorophyll content in crops. Geophysical Research Letters, v. 32, n. 8, p. L08403, 2005.

GITELSON, A.A., MERZLYAK, M.N. Signature analysis of leaf reflectance spectral: algorithm development for remote sensing of chlorophyll. Journal of Plant Physiology, v. 148, p. 494-500, 1996.

GOERGEN, L. C. G.; KILCA, R. de V.; NARVAES, I. da S.; SILVA, M. N.; SILVA, E. A.; PEREIRA, R. S.; ADAMI, M. Distinção de espécies de eucalipto de diferentes idades por meio de imagens TM/Landsat 5. Pesquisa Agropecuária Brasileira, v.51, n.1, p.53-60, 2016.

GOERGEN, L. C. G.; SOARES, J. C. W.; BERRA, E. F.; BRADELERO, C.; PEREIRA, R. S. Índices espectrais para avaliação da dinâmica sazonal de clorofilas em folhas de Eucalyptus grandis. Pesquisa Florestal Brasileira, v. 35, n. 82, p. 55-61, 2015.

GUYOT, G.; GUYON, D.; RIOM, J. Factors affecting the spectral response of forest canopies: a review. Geocarto International, v. 4, n. 3, p. 3-18, 1989.

INSTITUTO NACIONAL DE METROLOGIA (INMET). Disponível em: <http://www.inmet.gov.br/sonabra/pg_dspDado sCodigo_sim.php?QTgwMw>. Acesso em: 9 dez. 2015.

JACKSON, R.D., HUETE, A. R. Interpreting vegetation indices. Journal of Preventive Veterinary Medicine, v.11, p.185-200, 1991. 
KNIPLING, E. B. Physical and physiological basis for the reflectance visible and near infrared radiation from vegetation. Remote Sensing of Environment, v.1, n.3. p. 155-159, 1970.

LI, F.; MIAO, Y.; HENNIG, S. D.; GNYP, M. L.; CHEN, X.; JIA, L.; BARET, G. Evaluating hyperspectral vegetation indices for estimating nitrogen concentration of winter wheat at different growth stages. Precision Agriculture, v. 11, n. 4, p. 335-357, 2010.

LILLESAND, T.M.; KIEFER, R.W. Remote Sensing and image interpretation. 3. ed. New York: John Wiley \& Sons, 1994. 750 p.

LINDENMAIER, D. S; SOUZA, B. S. P. Geografia ensino \& pesquisa. Avaliação da cobertura vegetal arbórea em Cachoeira do Sul/RS: índice e distribuição espacial do elemento verde na paisagem urbana, v. 19, n. 3, p. 79-87, 2015.

LUCHIARI, A. Identificação da cobertura vegetal em áreas urbanas por meio de produtos de sensoriamento remoto e de um sistema de informação geográfica. Revista do Departamento de Geografia, v.14, p.47-58, 2001.

MILLER, J. R.; HARE, E. W.; WU, J. Quantitative characterization of the vegetation red edge reflectance 1. An inverted-Gaussian reflectance model. International Journal of Remote Sensing, v. 11, n. 10, p. 1755-1773, 1990.

MCCUNE, B.; MEFFORD, M. J. PC-ORD multivariate analysis of ecological data, version 6.0. MjM Software Design, Gleneden Beach, 2011.

MOREIRA, M. A. Fundamentos do sensoriamento remoto e metodologias de aplicação. 4 ed. atualizada e ampliada. Viçosa: UFV, 2011. 422p.

MOTOMIYA, A. V, A.; MOLIN, J. P.;
CHIAVEGATO, E. J. Utilização de sensor óptico ativo para detectar deficiência foliar de nitrogênio em algodoeiro. Revista Brasileira de Engenharia Agrícola e ambiental, v.13, n.2, p.137-145, 2009

PADWICK, C.; DESKEVICH, M.; PACIFICI, F.; SMALLWOOD, S. WorldView-e PanSharpening. In: ASPRS 2010 Annual Conference, 2010. Anais. San Diego, California, $2010 . \quad$ Disponível em: <http://info.asprs.org/publications/proceedings/ sandiego2010/sandiego10/Padwick.pdf >.

Acesso em: 10 nov 2015.

PARANHOS FILHO, A. C.; LASTORIA, G.; TORRES, T. G. Sensoriamento remoto ambiental aplicado: introdução às geotecnologias. Campo Grande, MS, UFMS. 198 p. 2008.

PASSO, D. P.; BIAS, E. S.; BRITES, R. S.; COSTA, G. A. O. P. Uso do sistema Interimage para identificação de alvos urbanos em imagens do satélite WordView II. Revista Brasileira de Cartografia, n.65/6, p. 1211-1221, 2013.

PAZ, C. R.; SAQUET, D. B.; FERRAZ, R. C.; PEREIRA, R. S.; COSTA, V. P. Discriminação de diferentes espécies de pastagens com uso da espectrorradiometria. XIV Simpósio Brasileiro de Sensoriamento Remoto, Anais. INPE, Natal, RN, 2009. p. 1175-1181.

PONZONI, F. J. Sensoriamento remoto da vegetação. 2. ed. Atualizada e ampliada, São Paulo: Oficina de Textos, 2012. 160p.

PONZONI, F.J.; SHIMABUKURO, Y.E. Diagnosticando a mata atlântica. In: PONZONI, J. F.; SHIMABUKURO, Y.E.; KUPLICH, T. M. Sensoriamento remoto no estudo da vegetação. 2. ed. Campos: INPE, 2007. São José dos Campos, SP: Parêntese, 2007. 127 p.

PONZONI, F.J. Comportamento espectral da vegetação. In.: MENESES, R.; MADEIRA NETTO, J.S. Sensoriamento remoto: 
refletância dos alvos naturais. Brasília: UnB, Planaltina: Embrapa Cerrados. p.157-199. 2001. PONZONI, F. J.; DISPERATI, A. A. Comportamento espectral da vegetação. São José dos Campos: INPE, 1995. 37 p.

RANGEL, M. E. S.; GURGEL, H. C.; Ferri, C.; SUGAWARA, L. M.; PINTO, M. L. A. Relação entre o Fator de Reflectância Hemisférica e o Fator de Reflectância Hemisférica Bidirecional de Folhas Isoladas da Tibouchina granulosa cogn. In: $\mathrm{X}$ Simpósio Brasileiro de Sensoriamento Remoto. Anais. INPE, Foz do Iguaçu, PR, 2001. p.1461-1467.

RIBEIRO, B. M. G.; FONSECA, L. M. F.; KUX, H. J. H. Mapeamento da cobertura do solo urbano utilizando imagens WorldView-ii e o sistema interimage. Revista Brasileira de Cartografia, n.63 (ed. Especial 40 anos), 2011.

SILVA E. L. S.; PONZONI, F. J. Comparação entre a reflectância hemisférica de folhas e a reflectância bidirecional de um dossel. Revista Árvore, v. 19, n. 4, p. 466-478, 1995.

SILVA, C. S., HELDWEIN, A. B., MARTINS, F. B., TRENTIN, G., \& GRIMM, E. L. Análise de distribuição de chuva para Santa Maria, RS. Revista Brasileira de Engenharia Agrícola e Ambiental, v. 11, p. 67-72. 2007.

SILVA, E. A.; MARANGON, G. P.; DESSBESELL, L.; MORAIS, W. W.; LIPPERT, D. B.; PEREIRA, R. S. CARACTERIZAÇÃO ESPECTRAL NA REFLECTÂNCIA DE Eucalyptus grandis. Revista Floresta, v. 42, n. 2, p. 285 - 292, 2012.
SHIMABUKURO, Y. E.; PONZONI, F.J. Sensor data applied to vegetation studies. Revista Brasileira de Cartografia, n.64/6, p. 873-866, 2012.

WANG, Z.X. et al. From AVHRR-NDVI to MODIS-EVI: advances in vegetation index research. Acta Ecologica Sinica, v. 23, n. 5, p. 979-988, 2003.

WATANABE, F. S. Y.; IMAI, N. N.; BOSCHI, L. S.; PITELLI, R. L. C. M. Caracterização de plantas aquáticas submersas a partir de dados de espectrorradiometro no reservatório de Nova Avanhandava - Rio Tietê/SP. In: XV Simpósio Brasileiro de Sensoriamento Remoto, Anais. INPE, Curitiba, PR, 2011. p. 8500-8507.

SCHRODER, T.; PEREIRA, R. S.; ZIMMERMANN, A. P. L. REDIN, C. G.; MACGADO, L. M.; FLECK, M. D.; ROCHA, N. S. Influência de métodos de coleta de dados espectrorradiométricos sob índices de vegetação em eucalipto. Revista Eletrônica em Gestão, Educação e Tecnologia Ambiental, v.19, n.3 p. 690-701, 2015. 